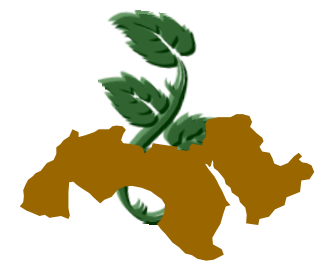

Arab Univ.

J. Agric. Sci., Ain Shams Univ., Cairo, 23(2), 475- 483, 2015

\title{
INFLUENCE OF MINERAL NITROGEN, COMPOST AND NITROGEN FIXING BACTERIA ON TOMATO PLANTS GROWN IN SANDY SOIL
}

\author{
Manal M.H. Gad El-Moula ${ }^{1}$ and S.A. Abou-El-Hassan ${ }^{2}$ \\ 1- Central Laboratory for Agricultural Climate, Agricultural Research Center, Giza, Egypt \\ 2- Central Lab of Organic Agriculture, Agricultural Research Center, Giza, Egypt \\ E-mail: Saad0777@yahoo.com
}

Keywords: Tomato, Mineral fertilization, Compost, Nitrogen fixing bacteria, Biofertilization

\section{ABSTRACT}

Pot trials were conducted under plastic house condition during two successive seasons of $2013 / 2014$ and $2014 / 2015$, at the experimental site of Central Laboratory for Agricultural Climate (CLAC), Agricultural Research Center, Giza, Egypt. The present study aims to determine the partial replacement of mineral nitrogen fertilization of tomato by nitrogen fixing bacteria with or without adding compost in sandy soil. Tomato seedlings (Lora $F_{1}$ Hybrid) were transplanted during the first week of October into plastic pots (30 $\mathrm{cm}$ diameter) filled with $10 \mathrm{~kg}$ of sandy soil. Three rates 25,50 and $75 \%$ of the recommended mineral nitrogen in the nutrient solution for tomato with adding compost at $2 \%$ and nitrogen fixing bacteria (Azotobacter chroococcum and Azospirillium brasilense) at $20 \mathrm{ml} /$ plant either individually or in combinations were investigated on growth, mineral composition and yield of tomato plants compared to $100 \%$ of recommended nitrogen only (control). The plants were irrigated daily by drip irrigation and received $200 \mathrm{ml} /$ plant of nutrient solution twice a weekly. The results showed that using 50 or $75 \%$ of $\mathrm{N}$ mineral fertilizer + compost + nitrogen fixing bacteria gave the highest values of growth, mineral composition and yield of tomato. It is recommended that $50 \%$ of nitrogen mineral fertilizers for tomato plants could be replaced by nitrogen fixing bacteria in presence of compost, which in earn, reduce environment pollution caused by extensive application of mineral nitrogen fertilizers.

\section{INTRODUCTION}

Tomato (Solanum lycopersicum) is one of the most popular and widely grown vegetable crops in the world. The total cultivated area in Egypt was 0.52 million feddans (feddan $=0.4$ hectare), produced about 8.6 million tons on annual basis with an average of productivity 16.636 tons/feddan (Ministry of Agriculture and Land Reclamation, 2013). The tomato crop is highly responsive to nitrogen fertilizer application, where the nitrogen availability may be limited factor for plant growth in many areas especially in low organic soils. (Taber, 2001).

Mineral nitrogen fertilizers often are easy dissolving and quickly leaching in soils, thus they can polluted soils and groundwater (Dhar, 1962). Excess use of nitrogen fertilizers deteriorates the soil health, increases ground water pollution, encourages nitrate accumulation in fruits and makes plants susceptible to pest and disease incidents (Chatterjee et al 2014). Therefore, management $\mathrm{N}$ fertilizer such as rate and type of $\mathrm{N}$ fertilizer is very important (De Pascale et al 2006). Thus, integrated nutrient management has become an accepted strategy to bring about improvement in soil fertility and protecting the environment. This strategy utilizes a judicious combination of inorganic, organic and bio fertilizers (Premsekhar and Rajashree, 2009).

In Egypt, the organic matter of cultivated clay soils is between 1.0- $2.5 \%$, while in the calcareous and sandy desert soils, it is usually less than $0.5 \%$ under arid and semiarid conditions (Abd ElGhaffar, 1982); so most of Egypt soils need to add organic amendments to improve their properties and consequently their productivity and natural fertility. The application of compost to these soils

(Received 12 May, 2015)

(Accepted 12 June, 2015) 
improve characteristics and fertility of soil and consequently increase the growth and development of plant roots, shoots and quantity of yield (Mamo et al 1998; Elashry et al 2008), as well as reduce the using of chemical fertilizers, which have adverse environmental effects (Mahmoud et al 2009).

Biological nitrogen fixation is one way of converting elemental nitrogen into plant usable form (Gothwal et al 2007). Nitrogen fixing bacteria (NFB) that function transform inert atmospheric nitrogen to organic compounds (Bakulin et al 2007). These bacteria provide the plant with fixed nitrogen, hormones, signal molecules, vitamins, iron, etc (Kavadia et al 2007; Mikhailouskaya and Bogdevitch, 2009). Azotobacter and Azospirillum are the two most important non-symbiotic $\mathrm{N}$ fixing bacteria in non-leguminous crops. Under appropriate conditions, Azotobacter and Azospirillum can enhance plant development and promote the yield of several agricultural important crops in different soils and climatic regions (Okon and Labendera-Gonzalez, 1994; Jagadeesha, 2008). Applying Nitrogen fixing bacteria is not only reducing mineral nitrogen requirements by $25 \%$, but also increases the availability of various nutrients, enhances the resistance of plants to root disease and reduces the environmental pollution (Rizk and Shafeek, 2000).

Many studies showed that the combination of biofertilizers with organic or chemical fertilizers further enhanced the growth, yield and quality of plants such as Togun and Akanbi (2003), Toor et al (2006); Fawzy et al (2007); Glala et al (2010); Habibi et al (2011); Glala et al (2012) and Glala et al (2013).
This work aims to the possibility of partial replacement of mineral nitrogen fertilization of tomato by nitrogen fixing bacteria with or without adding compost in sandy soil.

\section{MATERIALS AND METHODS}

The experiment was carried out at the experimental site of Central Laboratory for Agricultural Climate (CLAC), Agricultural Research Center, under plastic house conditions, during the two successive seasons of 2013/2014 and 2014/2015. Tomato seeds (Lora $F_{1}$ Hybrid) were sown in the nursery on 27 August and the seedlings were transplanted to plastic house on 7 October in the both seasons. The seeds were sown in the seedling trays, which were filled with peat moss and vermiculite $1: 1(\mathrm{v}: \mathrm{v})$. for 40 days. Then they were transplanted into plastic pots 1 seedling / pot (30 $\mathrm{cm}$ diameter and $25 \mathrm{~cm}$ height), the pots were filled with $10 \mathrm{~kg}$ of sandy soil. Each treatment included 12 plants in one row, the space within plants and between rows was $50 \mathrm{~cm}$, the space between double rows was $75 \mathrm{~cm}$. The plants were irrigated by drip irrigation ( $4 \mathrm{~L} / \mathrm{hr}$ ) daily according to water rations program for tomato plants under plastic houses at Giza Governorate (Ministry of Agriculture and Land Reclamation, 1988).

The compost was added to sand pots as soil amendment at rate $2 \%(200 \mathrm{~g} / \mathrm{pot})$ before one week from transplanting except control treatment, as $200 \mathrm{~g}$ of additional sand soil was added per pot. The analyses of soil and compost were carried out according Chapman and Pratt (1961) and were presented in Tables (1 and 2) respectively.

Table 1. Physical and chemical analyses of experimental soil

\begin{tabular}{|c|c|c|c|c|c|c|c|c|c|c|c|c|c|}
\hline \multirow{2}{*}{$\begin{array}{c}\text { Sand } \\
\%\end{array}$} & $\begin{array}{c}\text { Silt } \\
\%\end{array}$ & $\begin{array}{c}\text { Clay } \\
\%\end{array}$ & Texture & $\mathbf{p H}$ & $\begin{array}{c}\mathbf{E C} \\
\mathbf{d S} / \mathbf{m}\end{array}$ & \multicolumn{4}{|c|}{ Cations meq/l } & \multicolumn{4}{|c|}{ Anions meq/l } \\
\cline { 6 - 13 } & & & $\mathbf{C a}^{++}$ & $\mathbf{M g}^{++}$ & $\mathbf{K}^{+}$ & $\mathbf{N a}^{+}$ & $\mathbf{C O}_{3}=$ & $\mathbf{H C O}_{3}^{-}$ & $\mathbf{C l}^{-}$ & $\mathbf{S O}_{4}=$ \\
\hline 90.76 & 6.78 & 2.46 & Sandy & 7.74 & 1.13 & 2.58 & 1.24 & 1.82 & 3.94 & 1.14 & 1.92 & 3.38 & 3.25 \\
\hline
\end{tabular}

Table 2. Chemical analyses of compost

\begin{tabular}{|c|c|c|c|c|c|c|}
\hline $\mathrm{pH} 1: 5$ & $\begin{array}{c}\text { EC 1:10 } \\
\mathbf{d S} / \mathbf{m}\end{array}$ & $\begin{array}{c}\text { O.M } \\
(\%)\end{array}$ & $\begin{array}{c}\mathbf{C} / \mathbf{N} \\
\text { ratio }\end{array}$ & $\mathbf{N} \%$ & $\mathbf{P} \%$ & $\mathbf{K} \%$ \\
\hline 7.86 & 4.62 & 28.58 & 15.63 & 1.28 & 0.93 & 1.08 \\
\hline
\end{tabular}

Nitrogen fixing bacteria (Azotobacter chroococcum and Azospirillium brasilense) as pure local 
strains were kindly provided by Microbiology Dept. Soil, Water and Environment Research Institute, Agricultural Research Center. Soil application technique was carried out by using liquid culture of Ashby media at a rate of $20 \mathrm{ml} /$ plant $(1 \mathrm{ml}$ contains $10^{8}$ cell) according to Mashhoor et al (2002) after diluted by water without Chlorine at $1: 20$. Nitrogen fixing bacteria were applied to the soil surface beside plants at twice times, after 1 and 3 weeks from transplanting.

The plants received $200 \mathrm{ml} /$ plant of nutrient solution (Abou-Hadid et al 1989) twice a weekly. The electrical conductivity (EC) of the nutrient solution was maintained at $2.5 \mathrm{dS} / \mathrm{m}$; while $\mathrm{pH}$ was maintained at $5.5-6.5$ by using nitric and phosphoric acids (3:1 v/v) as described Abou-Hadid et al (1989). The contains of the nutrient solution were showed in Table (3).

Table 3. Contains of the nutrient solution

\begin{tabular}{|c|c|c|c|c|c|c|c|c|c|c|c|c|c|}
\hline \multirow{2}{*}{ pH } & \multirow{2}{*}{$\begin{array}{c}E C \\
\mathrm{dS} / \mathrm{m}\end{array}$} & \multicolumn{6}{|c|}{ Macronutrients (ppm) } & \multicolumn{6}{|c|}{ Micronutrients (ppm) } \\
\hline & & $\mathbf{N}$ & $\mathbf{P}$ & $\mathbf{K}$ & $\mathrm{Ca}$ & Mg & S & $\mathrm{Fe}$ & Mn & B & $\mathrm{Cu}$ & Mo & $\mathrm{Zn}$ \\
\hline 6.5 & 2.5 & 260 & 35 & 300 & 160 & 50 & 221 & 5.0 & 1.0 & 0.3 & 0.1 & 0.1 & 0.1 \\
\hline
\end{tabular}

Three rates 25, 50 and 75\% (65, 130 and 195 ppm respectively) of mineral nitrogen from the recommended dose in nutrient solution $(260 \mathrm{ppm})$ as $1.44 \mathrm{~g}$ calcium nitrate $(15.5 \% \mathrm{~N})$ and $0.84 \mathrm{~g}$ potassium nitrate $(13 \% N)$ / $L$ of water with adding compost and nitrogen fixing bacteria individually or in combinations were investigated for production of tomato comparing to $100 \%$ of mineral nitrogen from the recommended dose in nutrient solution for tomato according to Abou-Hadid et al (1989) as a control.

\section{The experimental treatments}

1. $100 \% \mathrm{~N}$ mineral fertilization in nutrient solution (260 ppm) as a control.

2. $25 \% \mathrm{~N}$ mineral fertilization in nutrient solution $(65 \mathrm{ppm})+$ compost

3. $50 \% \mathrm{~N}$ mineral fertilization in nutrient solution (130 ppm) + compost.

4. $75 \% \mathrm{~N}$ mineral fertilization in nutrient solution $(195 \mathrm{ppm})+$ compost.

5. $25 \% \mathrm{~N}$ mineral fertilization + Nitrogen fixing bacteria (NFB).

6. $50 \% \mathrm{~N}$ mineral fertilization + Nitrogen fixing bacteria (NFB).

7. $75 \% \mathrm{~N}$ mineral fertilization + Nitrogen fixing bacteria (NFB).

8. $25 \% \mathrm{~N}$ mineral fertilization+ compost + Nitrogen fixing bacteria.

9. $50 \% \mathrm{~N}$ mineral fertilization+ compost + Nitrogen fixing bacteria.

10. $75 \% \mathrm{~N}$ mineral fertilization + compost +Nitrogen fixing bacteria.
The experimental treatments were arranged in a completely randomized block design, with three replicates for each treatment. Each replicate was included 12 pots. Tomato plants were grown in plastic house on the main branch only with the removal of all side branches. Supported strings were wrapped around the plants weekly.

After 60 days from transplanting, three plants from each replicate were randomly chosen to measure plant height, stem diameter and number of leaves on plant, as well as chlorophyll reading in the fourth upper leaf was measured by using Minolta Chlorophyll Meter Spad 501.

Total nitrogen, phosphorous and potassium percent were determined in the dry matter of fourth upper leaf according to Cottenie et al (1982). Samples were dried at $70^{\circ} \mathrm{C}$ for 72 hours according to ADAS/MAFF (1987). Then dried leaves were digested in sulphuric acid and hydrogen peroxide according to FAO (1980). Total nitrogen was determined by Kjeldahl method according to the procedure described by FAO (1980). Phosphorus percent was determined using spectrophotometer according to Watanabe and Olsen (1965). Potassium percent was determined spectrometrically using Phillips Unicum Atomic Absorption Spectrometer as described by Chapman and Pratt (1961).

Fresh and dry shoot weight of tomato plants was measured at the end of harvesting stage. Total yield and number of fruits per plant were recorded after each harvesting accumulatively until the end of harvesting season. 
Five ripe fruits (fully red color) from the same cluster position and fruit position on cluster per replicate, were selected to measure fruit weight, also total soluble solids (TSS) were measured by using a manual Refractometer.

Data of the two seasons were arranged and statistically analyzed by the analysis of variance using one way ANOVA according to Snedecor and Cochran (1980) with SAS software, version 2004. Comparison of treatment means was done using Tukey test at significance level 0.05 .

\section{RESULTS AND DISCUSSION}

Data in Tables (4 and 5) show the effect of applying $\mathrm{N}$-mineral fertilizer, compost and $\mathrm{N}$-fixing bacteria on vegetative growth parameters, such data show clearly that, using 50 or $75 \%$ of $\mathrm{N}$ mineral fertilizer + compost $+\mathrm{N}$-fixing bacteria significantly increased all vegetative growth parameters (plant height, stem diameter, leaf number / plant, fresh and dry shoot weight). Also the same treatments reflected the highest reading of chlorophyll of plant leaf during both seasons of study. However, the lowest value was found in the treatment of $25 \% \mathrm{~N}$-mineral fertilizer + compost or $\mathrm{N}$ fixing bacteria. The same trend was found in the second season. The increases in plant growth obtained when partial replacement of mineral nitrogen by $\mathrm{N}$-fixing bacteria in presence of compost, might be due to the improvement of physical and chemical properties of soil by adding compost (Mamo et al 1998), which improve soil fertility and biological activity in roots rhizosphere (Glala et al 2010 and 2012). As well as, $\mathrm{N}$-fixing bacteria provide the plant with fixed nitrogen, hormones, signal molecules, vitamins, iron, etc which enhance root growth of plants (Kavadia et al 2007; Mikhailouskaya and Bogdevitch, 2009). All that, play an important role in increasing nutrient availability for uptake which reflected in better root distribution and vegetative growth. These results agreed with those obtained by Abdalla et al (2001) on pepper, Glala et al (2012), on squash, Glala et al (2010) and Glala et al (2013) on tomato plants.

Table 4. Effect of different treatments on plant height, stem diameter and leaves number of tomato plants (60 days after transplanting) during 2013/2014 and 2014/2015 seasons

\begin{tabular}{|c|c|c|c|c|c|c|}
\hline \multirow{2}{*}{ Treatments } & \multicolumn{2}{|c|}{$\begin{array}{c}\text { Plant height } \\
\text { cm }\end{array}$} & \multicolumn{2}{|c|}{$\begin{array}{c}\text { Stem diameter } \\
\mathrm{mm}\end{array}$} & \multicolumn{2}{|c|}{ Leaf No./plant } \\
\hline & $\begin{array}{c}1^{\text {st }} \\
\text { season }\end{array}$ & $\begin{array}{c}2^{\text {nd }} \\
\text { season }\end{array}$ & $\begin{array}{c}1^{\text {st }} \\
\text { season }\end{array}$ & $\begin{array}{c}2^{\text {nd }} \\
\text { season }\end{array}$ & $\begin{array}{c}1^{\text {st }} \\
\text { season }\end{array}$ & $\begin{array}{c}2^{\text {nd }} \\
\text { season }\end{array}$ \\
\hline $100 \% \mathrm{NM}$ (control) & $193.83 \mathrm{~b}$ & $200.42 b$ & $19.67 b$ & $20.00 \mathrm{~b}$ & $29.33 b$ & $30.67 b$ \\
\hline $25 \% N M+C$ & $161.67 \mathrm{~d}$ & $166.29 \mathrm{e}$ & $13.33 \mathrm{fg}$ & $13.33 \mathrm{f}$ & $19.67 \mathrm{e}$ & 20.33 ef \\
\hline $50 \% N M+C$ & $175.00 \mathrm{c}$ & $180.24 d$ & 15.00 def & $15.67 \mathrm{de}$ & $23.33 d$ & $24.00 \mathrm{~cd}$ \\
\hline $75 \% N M+C$ & $183.33 \mathrm{bc}$ & $190.86 \mathrm{c}$ & $16.67 \mathrm{~cd}$ & $17.33 \mathrm{~cd}$ & $24.33 \mathrm{~cd}$ & $26.00 \mathrm{~cd}$ \\
\hline $25 \% \mathrm{NM}+\mathrm{NFB}$ & $158.33 \mathrm{~d}$ & $164.63 \mathrm{e}$ & $13.00 \mathrm{~g}$ & $13.00 \mathrm{f}$ & $19.00 \mathrm{e}$ & $19.33 \mathrm{f}$ \\
\hline $50 \% \mathrm{NM}+\mathrm{NFB}$ & $174.67 \mathrm{c}$ & $179.60 \mathrm{~d}$ & $14.67 \mathrm{efg}$ & $15.33 \mathrm{e}$ & $22.67 \mathrm{~d}$ & $23.67 \mathrm{de}$ \\
\hline $75 \% \mathrm{NM}+\mathrm{NFB}$ & $181.33 \mathrm{c}$ & $187.50 \mathrm{~cd}$ & $16.33 \mathrm{cde}$ & $17.00 \mathrm{cde}$ & $23.67 \mathrm{~cd}$ & $25.33 \mathrm{~cd}$ \\
\hline $25 \% N M+C+N F B$ & $185.28 \mathrm{bc}$ & $192.88 \mathrm{bc}$ & $17.33 \mathrm{c}$ & $18.00 \mathrm{c}$ & $26.33 c$ & $27.33 \mathrm{bc}$ \\
\hline $50 \% N M+C+N F B$ & $211.73 \mathrm{a}$ & $216.44 \mathrm{a}$ & $22.33 \mathrm{a}$ & $22.67 \mathrm{a}$ & $33.33 \mathrm{a}$ & $34.33 \mathrm{a}$ \\
\hline $75 \% N M+C+N F B$ & $214.17 \mathrm{a}$ & $221.45 \mathrm{a}$ & $23.00 \mathrm{a}$ & $23.00 \mathrm{a}$ & $34.00 \mathrm{a}$ & $35.00 \mathrm{a}$ \\
\hline
\end{tabular}

Means followed in same column by similar letters are not statistically different at 0.05 level according to Tukey test.

$\mathrm{NM}=$ mineral nitrogen fertilizer $\quad \mathrm{C}=$ compost

$\mathrm{NFB}=$ nitrogen fixing bacteria (Azotobacter chroococcum and Azospirillium brasilense) 
Table 5. Effect of different treatments on fresh, dry weight and chlorophyll reading of tomato plants during 2013/2014 and 2014/2015 seasons

\begin{tabular}{|c|c|c|c|c|c|c|}
\hline \multirow{2}{*}{ Treatments } & \multicolumn{2}{|c|}{$\begin{array}{l}\text { Fresh weight } \\
\text { kg/plant }\end{array}$} & \multicolumn{2}{|c|}{$\begin{array}{l}\text { Dry weight } \\
\text { q/plant }\end{array}$} & \multicolumn{2}{|c|}{$\begin{array}{c}\text { Chlorophyll reading } \\
\text { Spad }\end{array}$} \\
\hline & $\begin{array}{c}1^{\text {st }} \\
\text { season }\end{array}$ & $\begin{array}{c}2^{\text {nd }} \\
\text { season }\end{array}$ & $\begin{array}{c}1^{\text {st }} \\
\text { season }\end{array}$ & $\begin{array}{c}2^{\text {nd }} \\
\text { season }\end{array}$ & $\begin{array}{c}1^{\text {st }} \\
\text { season }\end{array}$ & $\begin{array}{c}2^{\text {nd }} \\
\text { season }\end{array}$ \\
\hline $100 \% \mathrm{NM}$ (control) & $1.35 \mathrm{a}$ & $1.43 \mathrm{~b}$ & $259.67 \mathrm{~b}$ & $262.00 \mathrm{~b}$ & $32.00 \mathrm{~b}$ & $32.82 \mathrm{~b}$ \\
\hline $25 \% N M+C$ & $1.16 \mathrm{f}$ & $1.22 \mathrm{e}$ & $216.33 \mathrm{f}$ & $217.67 \mathrm{f}$ & $22.67 \mathrm{e}$ & $23.50 \mathrm{e}$ \\
\hline $50 \% N M+C$ & $1.23 \mathrm{de}$ & $1.27 \mathrm{~d}$ & $224.33 \mathrm{e}$ & $226.33 \mathrm{e}$ & $25.67 d$ & $27.13 \mathrm{~d}$ \\
\hline $75 \% N M+C$ & $1.27 \mathrm{~cd}$ & $1.34 \mathrm{c}$ & $233.67 d$ & $236.00 \mathrm{~d}$ & $27.67 \mathrm{~cd}$ & $30.35 \mathrm{bc}$ \\
\hline $25 \% N M+N F B$ & $1.16 \mathrm{f}$ & $1.21 \mathrm{e}$ & $215.67 f$ & $217.67 \mathrm{f}$ & $22.33 \mathrm{e}$ & $23.16 \mathrm{e}$ \\
\hline $50 \% \mathrm{NM}+\mathrm{NFB}$ & $1.21 \mathrm{ef}$ & $1.28 \mathrm{~d}$ & $223.33 \mathrm{e}$ & $225.33 \mathrm{e}$ & $25.67 d$ & $27.13 d$ \\
\hline $75 \% \mathrm{NM}+\mathrm{NFB}$ & 1.25 cde & $1.33 \mathrm{c}$ & $233.33 d$ & $236.33 d$ & $27.67 \mathrm{~cd}$ & $29.69 \mathrm{~cd}$ \\
\hline $25 \% N M+C+N F B$ & $1.29 \mathrm{bc}$ & $1.36 \mathrm{c}$ & $240.67 \mathrm{c}$ & $243.00 \mathrm{c}$ & $28.33 \mathrm{c}$ & $29.95 \mathrm{~cd}$ \\
\hline $50 \% N M+C+N F B$ & $1.59 \mathrm{a}$ & $1.69 \mathrm{a}$ & $287.67 \mathrm{a}$ & $289.67 \mathrm{a}$ & $35.00 \mathrm{a}$ & $36.21 \mathrm{a}$ \\
\hline $75 \% N M+C+N F B$ & $1.61 \mathrm{a}$ & $1.70 \mathrm{a}$ & $289.00 \mathrm{a}$ & $290.33 \mathrm{a}$ & $35.67 \mathrm{a}$ & $36.30 \mathrm{a}$ \\
\hline
\end{tabular}

Means followed in same column by similar letters are not statistically different at 0.05 level according to Tukey test. $\mathrm{NM}=$ mineral nitrogen fertilizer $\quad \mathrm{C}=$ compost

NFB $=$ nitrogen fixing bacteria (Azotobacter chroococcum and Azospirillium brasilense)

Data in Table (6) revealed that the highest concentrations of $\mathrm{N}, \mathrm{P}$ and $\mathrm{K}$ were obtained by $50 \%$ or $75 \% \mathrm{~N}$-mineral fertilizer + compost $+\mathrm{N}$ fixing bacteria treatments. On the other hand, applying $25 \% \mathrm{~N}$-mineral fertilizer + compost or $\mathrm{N}$ fixing bacteria gave the lowest concentrations. These results were similar in both seasons. These findings might be due to the beneficial effects of $\mathrm{N}$ fixing bacteria that help in increasing nitrogen fixation and other nutrients in rhizosphere, also enhance the production of phytohormone (Kavadia et al 2007; Mikhailouskaya and Bogdevitch, 2009). Moreover, compost has a high cation exchange capacity exceeded the capacity of sandy soil to maintain nutrients are absorbed by plants. Consequently, root system absorbs more nutrients (Togun and Akanbi, 2003; Toor et al 2006; Ahmad et al 2008; Fiorentino and Fagnano, 2011; Abou-El-Hassan et al 2014). All that lead to increment minerals content of plants.

The results in Table (7) demonstrate clearly that, using $\mathrm{N}$-mineral fertilizer, compost and $\mathrm{N}$ fixing bacteria affected significantly on number and weight of fruit / plant and TSS in tomato fruits. The highest values of yield, fruit number and TSS were found by $50 \%$ or $75 \% \mathrm{~N}$-mineral fertilizer +compost $+\mathrm{N}$-fixing bacteria treatments. On the contrary, the lowest values were recorded by the treatments of $25 \% \mathrm{~N}$-mineral fertilizer + compost or $\mathrm{N}$-fixing bacteria individually. Meanwhile, the other treatments were moderated. These results were true in the two seasons. This increment in the yield and quality of tomato fruits, may be resulted to positive effect of compost in sandy soil, which improve the soil characteristics and fertility consequently increase the growth and development of plant roots (Mamo et al 1998 and Elashry et al 2008). As well as, $\mathrm{N}$-fixing bacteria have beneficial effects such as increasing nitrogen fixation and other nutrients in rhizosphere, also production of phytohormone that improve root development and increase the rate of water and mineral uptake by roots (Okon and Labendera-Gonzalez, 1994; Jagadeesha, 2008). All these factors combined together produce better nutrients absorption of root, which reflects better vegetative growth, photosynthetic activity and dry matter accumulation. which lead to produce good yield and quality of tomato. These results agreed with those obtained by Abdalla et al (2001) on pepper, Habibi et al (2011), on pumpkin, Glala et al (2012), on squash, Glala et al (2010) and Glala et al (2013) on tomato. 
Table 6. Effect of different treatments on NPK percent of tomato plants during 2013/2014 and 2014/2015 seasons

\begin{tabular}{|c|l|l|l|l|l|l|}
\hline \multirow{2}{*}{ Treatments } & \multicolumn{2}{|c|}{$\mathbf{N}$} & \multicolumn{1}{c|}{$\mathbf{P}$} & \multicolumn{2}{c|}{ K } \\
\cline { 2 - 7 } & \multicolumn{1}{|c|}{$\begin{array}{c}\mathbf{1}^{\text {st }} \\
\text { season }\end{array}$} & $\begin{array}{c}\mathbf{2}^{\text {nd }} \\
\text { season }\end{array}$ & $\begin{array}{c}\mathbf{1}^{\text {st }} \\
\text { season } \\
\text { season }\end{array}$ & $\begin{array}{c}\mathbf{1}^{\text {st }} \\
\text { season }\end{array}$ & $\begin{array}{c}\mathbf{2}^{\text {nd }} \\
\text { season }\end{array}$ \\
\hline $100 \% \mathrm{NM}$ (control) & $3.66 \mathrm{bc}$ & $3.81 \mathrm{~b}$ & $0.673 \mathrm{~b}$ & $0.695 \mathrm{~b}$ & $4.37 \mathrm{~b}$ & $4.52 \mathrm{~b}$ \\
$25 \% \mathrm{NM}+\mathrm{C}$ & $2.79 \mathrm{e}$ & $2.87 \mathrm{~d}$ & 0.320 ef & $0.334 \mathrm{f}$ & 3.55 ef & 3.64 ef \\
$50 \% \mathrm{NM}+\mathrm{C}$ & $3.01 \mathrm{de}$ & $3.11 \mathrm{~cd}$ & $0.377 \mathrm{e}$ & $0.392 \mathrm{e}$ & $3.73 \mathrm{de}$ & $3.83 \mathrm{de}$ \\
$75 \% \mathrm{NM}+\mathrm{C}$ & $3.24 \mathrm{cde}$ & $3.33 \mathrm{bcd}$ & $0.457 \mathrm{~d}$ & $0.474 \mathrm{~d}$ & $3.97 \mathrm{~cd}$ & $4.10 \mathrm{~cd}$ \\
$25 \% \mathrm{NM}+\mathrm{NFB}$ & $2.77 \mathrm{e}$ & $2.86 \mathrm{~d}$ & $0.303 \mathrm{f}$ & $0.316 \mathrm{f}$ & $3.35 \mathrm{f}$ & $3.43 \mathrm{f}$ \\
$50 \% \mathrm{NM}+\mathrm{NFB}$ & $3.00 \mathrm{de}$ & $3.09 \mathrm{~cd}$ & $0.373 \mathrm{e}$ & $0.391 \mathrm{e}$ & $3.37 \mathrm{ef}$ & $3.45 \mathrm{ef}$ \\
$75 \% \mathrm{NM}+\mathrm{NFB}$ & $2.90 \mathrm{de}$ & $2.98 \mathrm{~cd}$ & $0.443 \mathrm{~d}$ & $0.466 \mathrm{~d}$ & $3.59 \mathrm{ef}$ & 3.71 ef \\
$25 \% \mathrm{NM}+\mathrm{C}+\mathrm{NFB}$ & $3.43 \mathrm{dc}$ & $3.43 \mathrm{bc}$ & $0.533 \mathrm{c}$ & $0.545 \mathrm{c}$ & $4.22 \mathrm{bc}$ & $4.35 \mathrm{bc}$ \\
$50 \% \mathrm{NM}+\mathrm{C}+\mathrm{NFB}$ & $4.17 \mathrm{a}$ & $4.37 \mathrm{a}$ & $0.910 \mathrm{a}$ & $0.954 \mathrm{a}$ & $4.77 \mathrm{a}$ & $4.90 \mathrm{a}$ \\
$75 \% \mathrm{NM}+\mathrm{C}+\mathrm{NFB}$ & $4.24 \mathrm{a}$ & $4.38 \mathrm{a}$ & $0.927 \mathrm{a}$ & $0.965 \mathrm{a}$ & $4.83 \mathrm{a}$ & $4.92 \mathrm{a}$ \\
\hline
\end{tabular}

Means followed in same column by similar letters are not statistically different at 0.05 level according to Tukey test. $\mathrm{NM}=$ mineral nitrogen fertilizer $\quad \mathrm{C}=$ compost

$\mathrm{NFB}=$ nitrogen fixing bacteria (Azotobacter chroococcum and Azospirillium brasilense)

Table 7. Effect of different treatments on yield, fruit No, fruit weight and TSS of tomato fruits during 2013/2014 and 2014/2015 seasons

\begin{tabular}{|c|c|c|c|c|c|c|c|c|}
\hline \multirow{2}{*}{ Treatments } & \multicolumn{2}{|c|}{$\begin{array}{c}\text { Yield } \\
\text { kg/plant }\end{array}$} & \multicolumn{2}{c|}{ Fruit No } & \multicolumn{2}{c|}{$\begin{array}{c}\text { Fruit weight } \\
\mathbf{g}\end{array}$} & \multicolumn{2}{c|}{$\begin{array}{c}\text { TSS } \\
\%\end{array}$} \\
\cline { 2 - 9 } & $\begin{array}{c}\mathbf{1}^{\text {st }} \\
\text { season }\end{array}$ & $\begin{array}{c}\mathbf{2}^{\text {nd }} \\
\text { season }\end{array}$ & $\begin{array}{c}\mathbf{1}^{\text {st }} \\
\text { season }\end{array}$ & $\begin{array}{c}\mathbf{2}^{\text {nd }} \\
\text { season }\end{array}$ & $\begin{array}{c}\mathbf{1}^{\text {st }} \\
\text { season }\end{array}$ & $\begin{array}{c}\mathbf{2}^{\text {nd }} \\
\text { season }\end{array}$ & $\begin{array}{c}\mathbf{1}^{\text {st }} \\
\text { season }\end{array}$ & $\begin{array}{c}\mathbf{2}^{\text {nd }} \\
\text { season }\end{array}$ \\
\hline $100 \% \mathrm{NM}$ (control) & $6.21 \mathrm{~b}$ & $6.54 \mathrm{~b}$ & $48.18 \mathrm{bc}$ & $48.00 \mathrm{~b}$ & $132.67 \mathrm{~b}$ & $137.05 \mathrm{~b}$ & $5.25 \mathrm{a}$ & $5.13 \mathrm{ab}$ \\
$25 \% \mathrm{NM}+\mathrm{C}$ & $4.43 \mathrm{f}$ & $4.58 \mathrm{f}$ & $41.83 \mathrm{e}$ & $42.00 \mathrm{~d}$ & $97.67 \mathrm{e}$ & $101.51 \mathrm{e}$ & $3.63 \mathrm{de}$ & $3.80 \mathrm{~d}$ \\
$50 \% \mathrm{NM}+\mathrm{C}$ & $5.22 \mathrm{e}$ & $5.49 \mathrm{e}$ & $44.49 \mathrm{~d}$ & $44.33 \mathrm{c}$ & $113.33 \mathrm{~d}$ & $116.41 \mathrm{~d}$ & $3.87 \mathrm{c}$ & $4.13 \mathrm{~cd}$ \\
$75 \% \mathrm{NM}+\mathrm{C}$ & $5.60 \mathrm{~d}$ & $5.84 \mathrm{~d}$ & $45.33 \mathrm{~d}$ & $45.33 \mathrm{c}$ & $123.33 \mathrm{c}$ & $127.41 \mathrm{c}$ & $3.97 \mathrm{c}$ & $4.33 \mathrm{~cd}$ \\
$25 \% \mathrm{NM}+\mathrm{NFB}$ & $4.42 \mathrm{f}$ & $4.57 \mathrm{f}$ & $41.43 \mathrm{e}$ & $41.67 \mathrm{~d}$ & $96.33 \mathrm{e}$ & $99.79 \mathrm{e}$ & $3.57 \mathrm{e}$ & $3.73 \mathrm{~d}$ \\
$50 \% \mathrm{NM}+\mathrm{NFB}$ & $5.20 \mathrm{e}$ & $5.48 \mathrm{e}$ & $44.41 \mathrm{~d}$ & $44.00 \mathrm{c}$ & $112.67 \mathrm{~d}$ & $115.79 \mathrm{~d}$ & $3.80 \mathrm{~cd}$ & $4.07 \mathrm{~cd}$ \\
$75 \% \mathrm{NM}+\mathrm{NFB}$ & $5.60 \mathrm{~d}$ & $5.90 \mathrm{~d}$ & $45.18 \mathrm{~d}$ & $45.00 \mathrm{c}$ & $123.00 \mathrm{c}$ & $127.06 \mathrm{c}$ & $3.93 \mathrm{c}$ & $4.27 \mathrm{~cd}$ \\
$25 \% \mathrm{NM}+\mathrm{C}+\mathrm{NFB}$ & $5.75 \mathrm{c}$ & $6.16 \mathrm{c}$ & $45.67 \mathrm{~cd}$ & $45.67 \mathrm{a}$ & $126.67 \mathrm{c}$ & $132.97 \mathrm{~b}$ & $4.53 \mathrm{~b}$ & $4.67 \mathrm{bc}$ \\
$50 \% \mathrm{NM}+\mathrm{C}+\mathrm{NFB}$ & $6.64 \mathrm{a}$ & $6.75 \mathrm{a}$ & $48.81 \mathrm{a}$ & $50.67 \mathrm{a}$ & $142.67 \mathrm{a}$ & $151.28 \mathrm{a}$ & $5.38 \mathrm{a}$ & $5.70 \mathrm{a}$ \\
$75 \% \mathrm{NM}+\mathrm{C}+\mathrm{NFB}$ & $6.71 \mathrm{a}$ & $6.83 \mathrm{a}$ & $49.53 \mathrm{a}$ & 51.33 & $143.33 \mathrm{a}$ & $152.62 \mathrm{a}$ & $5.45 \mathrm{a}$ & $5.77 \mathrm{a}$ \\
\hline
\end{tabular}

Means followed in same column by similar letters are not statistically different at 0.05 level according to Tukey test.

$\mathrm{NM}=$ mineral nitrogen fertilizer $\quad \mathrm{C}=$ compost

$\mathrm{NFB}=$ nitrogen fixing bacteria (Azotobacter chroococcum and Azospirillium brasilense) 


\section{CONCLUSION}

It is clear from results of this study that $50 \%$ of nitrogen requirement for tomato plants as mineral fertilizers could be replaced by nitrogen fixing bacteria of Azotobacter chroococcum and Azospirillium brasilense in presence of compost by rate $2 \%$, that improved the use efficiency of nitrogen fertilizers and reduced the environment pollution caused by extensive application of mineral fertilizers.

\section{ACKNOWLEDGEMENT}

This work has been supported by Central Laboratory for Agricultural Climate (CLAC) and Central Lab of Organic Agriculture (CLOA), Agricultural Research Center, Giza, Egypt.

\section{REFERANCES}

Abdalla, A.M., Rizk, F.A. and Adam, S.M. 2001. The productivity of pepper plants as influenced by some Biofertilized treatments under plastic house condition. Bull Fac. Agric., Cairo Univ., 52: 625-640.

Abd El-Ghaffar, A.S. 1982. The significance of organic materials to Egyptian agriculture and maintenance of soil productivity. FAO Soil Bulletin, Rome, Italy, 45: 15-20.

Abou-Hadid, A.F., Zayed, A.M., El-Behairy, U.A. and El-Beltagy, A.S. 1989. A comparison between nutrient film technique (NFT) and soil for tomato production under protect cultivation in Egypt. Egypt. J. Hort. 16(2): 111-118.

Abou-El-Hassan S., Abdrabbo, M.A.A. and Desoky, A.H. 2014. Enhancing organic production of cucumber by using plant growth promoting rhizobacteria and compost tea under sandy soil condition. Res. J. Agric. \& Biol. Sci., 10(2):162-169.

ADAS/MAFF, 1987. The Analysis of Agricultural Material Book. 427, $3^{\text {rd }}$ Edition HMSO. London.

Ahmad, R., Shehzad, S.M., Khalid, A., Arshad, M. and Mahmood, M.H. 2008. Growth and yield response of wheat and maize to nitrogen and $\mathrm{L}$ tryptophan enriched compost. Pak. J. Bot., 39(2): 541-549.

Bakulin, M.K., Grudtsyna, A.S. and Pletneva, A. 2007. Biological fixation of nitrogen and growth of bacteria of the genus Azotobacter in liquid media in the presence of Perfluorocarbons. Appl. Biochem. Microbiol., 4: 399-402.
Chapman, H.D. and Pratt, P.F. 1961. Methods of Analysis for Soil. Plant and Water Division of Agric. Sci., Calif. Univ.

Chatterjee, R., Bandyopadhyay, S. and Jana, J.C. 2014. Impact of Organic Amendments and Inorganic fertilizers on production potential, nitrogen use efficiency and nitrogen balance in tomato. International Journal of Scientific Research in Knowledge, 2(5): 233-240.

Cottenie, A., Verloo, M., Kiekers, L., Velghe, G. and Camrbynek, R. 1982. Chemical Analysis of Plants and Soils. Hand Book, 1-63, Ghent, Belgium.

De Pascale, S., Tamburrino, R., Maggio, A., Barbieri, G., Fogliano, V. and Pernice, R. 2006. Effect of nitrogen fertilization on the nutritional value of organically and conventionally grown tomatoes. Acta Hort., 700: 107-110.

Dhar, N.R. 1962. Nitrogen fixation by organic matter in soil improvement. J. Indian Soc, soil Sci., 10: 76-96.

Elashry, S.M., Hanan, S. and Abd El-Moez, M.R. 2008. Ameliorative effect of organic fertilizers in the presence of mineral fertilizers on lettuce and sorghum yield and their components. Aust. J. Basic Appl. Sci., 2(4): 1246-1257.

FAO (Food and Agriculture Organization) 1980. Soil and Plant Analysis. Soils Bulletin 38/2,250.

Fawzy, Z.F., El-Bassiony and Saleh, S.A. 2007. Effect of chemical fertilizer, poultry manure and biofertilizer on growth,yield and chemical contents of tomato plants. J. Agric. Sci. Mansoura Univ., 32(8): 6583-6594.

Fiorentino, N. and Fagnano, M. 2011. Soil fertilization with composted solid waste: short term effects on lettuce production and mineral $\mathrm{N}$ availability. Geophysical Research Abstracts, Vol. 13, p 10520.

Glala A.A., Marzouk, N.M., Al-Bassyuni, M.S.S. and Hassan, N.M.K. 2013. Influence of Organic Nitrogen Fertilizers Replacement Rates Associated with Azosprillum spp, Enrichment on Tomato. J. Appl. Sci. Res., 9(3): 1952-1959.

Glala, A.A., Abd El-Samad, E.E.H., El-Abd, S.O. and Obiadalla-Ali, H.A. 2012. Increasing Organic Production of Summer Squash by Modulating Plant Sex Ratio. Acta Hort. (ISHS), 933: 137-143.

Glala, A.A., Ezzo, M.I. and Abd-Alla, A.M. 2010. Influence of Plant Growth Promotion Rhizosphere-Bacteria "PGPR" Enrichment and Some Alternative Nitrogen Organic Sources on Tomato. Acta Hort., 852: 131-138. 
Gothwal, R.K., Nigam, V.K., Mohan, M.K., Sasmal, D. and Ghosh, P. 2007. Screening of nitrogen fixers from rhizospheric bacterial isolates associated with important desert plants. Appl. Ecol. Environ. Res., 6(2): 101-109.

Habibi, A., Heidari, G., Sohrabi, Y., Badakhshan, H. and Mohammadi, K. 2011. Influence of bio, organic and chemical fertilizers on medicinal pumpkin traits. J. Med. Plants Res., 5(23): 5590-5597.

Jagadeesha, V. 2008. Effect of organic manure and biofertilizers on growth seed yield and quality in tomato cv. Megha. M.Sc. Thesis Fac. Agric., Dharwad Univ., India.

Kavadia, A., Vayenas, DV., Pavlou, S. and Aggelis, G. 2007. Dynamics of free living nitrogen fixing bacterial population in antagonistic conditions. Ecol. Model, 200: 243-253.

Mahmoud, E., Abd El-Kader, N., Robin, P., Akkal-Corfini, N. and Abd El-Rahman, L. 2009. Effects of different organic and inorganic fertilizers on cucumber yield and some soil properties. World J. Agric. Sci., 5 (4): 408-414.

Mamo, M., Rosen, C.J., Hallbach, T.R. and Moncrief, J.F. 1998. Corn yield and nitrogen uptake in sandy soils amended with municipal solid waste compost. J. Pro. Agri., 11: 469475.

Mashhoor, W.A., El-Borollosy, M.A., AbdelAzeem, H.H.A., Nasr, S.A. and Selim, S.M. 2002. Biofertilization of wheat plants exposed to environmental conditions. J. Agric. Sci, Ain Shams Univ., 10(2): 543-565.

Mikhailouskaya, N. and Bogdevitch, I. 2009. Effect of biofertilizers on yield and quality of long- fibred flax and cereal grains. J. Agron. Res., 7: 412-418.

Ministry of Agriculture and Land Reclamation (EAS), Economic Affairs Sector 2013. Bulletin of Agriculture Statistics.

Ministry of Agriculture and Land Reclamation, 1988. Infrastructure and Irrigation Systems for Protected Crops. Bulletin No. 2: 51-57.
Okon, Y. and Labandera-Gonzalez, C. 1994. Agronomic applications of Azospirillum: an evaluation of 20 years worldwide field inoculation. Soil Biol. Biochem., 26: 1591-1601.

Premsekhar, M. and Rajashree, V. 2009. Influence of bio-fertilizers on the growth characters, yield attributes, yield and quality of tomato. American-Eurasian J. Sustain. Agric., 3(1): 68-70.

Rizk, F.A. and Shafeek, M.R. 2000. Response of growth and yield of Vicia faba to foliar and bio fertilizers. Egypt . J. APPL. Sci., 15(12): 652670.

Snedecor, G.W. and Cochran, W.G. 1980. Statistical methods. Sixth Edition, lowa state university press, Ames., lowa, U.S.A.

Taber, H.G. 2001. Petiole sap nitrate sufficiency values for fresh market tomato production. J. Plant Nutr., 24: 945-959.

Togun, A.O. and Akanbi, W.B. 2003. Comparative effectiveness of organic based fertilizer to mineral fertilizer on tomato growth and fruit yield. Compost Science and Utilization, 11(4): 337-342.

Toor, R.K., Savage, G.P. and Heeb, A. 2006. Influence of different types of fertilizers on the major antioxidant components of tomatoes. Journal of Food Composition and Analysis, 19(1): 20-27.

Watanabe, F.S. and Olsen, S.R. 1965. Test of an ascorbic acid method for determining phosphorus in water and $\mathrm{Na} \mathrm{HCO} 3$ extracts from soil. Soil Sci. Soc. Amer. Proc., 29: 677-678.

Zaki, M.F., Hamouda, H.A., Ahmed, A.A. and El-bassiony, M.S. 2009. Integrated effect between phosphate dissolving bacteria and different phosphorus rates on the growth, productivity and quality of tomato cultivars. Egypt. J. Appl. Sci., 24(10A): 271-292. 